\title{
Zoophilic recidivism in schizophrenia: a case report
}

Zoophilia is a disorder of sexual preference characterized by recurrent, intense, sexually arousing fantasies, urges, or behaviours involving animals. ${ }^{1}$ Sexual contact with animals often occurs as a substitute for heterosexual relations when the animal is more readily accessible than a human partner. ${ }^{2}$ It may also be the result of curiosity or desire for novelty. In rare cases, however, Zoophilia becomes the principal form of sexual arousal and precludes the formation of normal sexual relationships. ${ }^{2}$ Although patients with schizophrenia make up a relatively small proportion of sex offenders, this small group may present significant clinical and legal challenges. ${ }^{3}$

The relationship between paraphilias and other mental disorder is complex and poorly understood. Though case reports of Zoophilia exist in the literature ${ }^{4,5}$, to the best of our knowledge, its occurrence and relationship with mental illness in Sub-Saharan Africa has not been reported.

We present a case report which highlights the psychodynamic and contextual issues involved in zoophilic recidivism in a person with schizophrenia. The Research and Ethical Committee of Neuropsychiatric Hospital Aro, Abeokuta, Nigeria and the legal custodian of the patient consented to publication of the case content.

Mr. A, a 28 year old single, unemployed male with incomplete secondary school education, was referred for psychiatric evaluation by the court following repeated sexual intercourse with animals. About 3 months before this presentation, he was found having intercourse with a pregnant goat which subsequently bled to death. Though he admitted to committing the sexual offence, he refused to elaborate on his reasons for doing so. He was neither under the control of external agents nor acting on hallucinatory commands. Prior to this incident, he had been observed fondling and walking the streets with goats. A few months earlier, Mr. A. had been observed talking and laughing to himself, he had become unduly aggressive towards others, threatened to burn his family house, his hygiene had deteriorated significantly, he began retaining used food wrappers in his room and refused to participate in household chores. He subsequently withdrew from people and became inappropriately close to animals.

About four years before the violent sexual offence, Mr. A. had received treatment for schizophrenia in a psychiatric facility. Though he remitted fully during inpatient treatment, there was medication non-adherence post-discharge.

Mr. A. is the second child in a monogamous family of 5 children. His early childhood years were marked by recurrent violent quarrels between his parents culminating in their separation when he was 10 years old. He continued residing with his father whom he described as harsh, authoritative and domineering. He yearned for his mother whom had been very protective and close to him During adolescence, he became truant and rebellious, adjusting poorly academically and sexually. Mr. A.'s relationship with animals dates back to this time when he was observed to be unusually attached to pet dogs. Many of his pets died in curious circumstances. Throughout adulthood he had no intimate relationships with females He was described as a stubborn individual, with a low threshold for violence, who preferred solitude and enjoyed watching films and keeping pets.

A mental state examination showed Mr. A to be alert, calm and cooperative. His speech was incoherent. He had euthymic mood but blunted affect, formal thought disorder (derailment) and persecutory delusions. There was no cognitive impairment and physical examination was normal. Though his symptoms resolved with antipsychotic medication, Mr. A's zoophilic interests persisted. He engaged in internet browsing for websites with animal related issues and was unusually interested in preparing animal food during occupational therapy activities. Mr. A. remained mentally stable at discharge and is on follow up and surveillance by the clinical team.

According to ICD-10 criteria, this patient can be diagnosed with Schizophrenia and Zoophilia (other disorder of sexual preference). As observed in a survey of persons with Zoophilia, most begin during adolescence. ${ }^{6}$ This case report supports that finding.

Mr. A's Zoophilic interests, commencing in adolescence, may initially have been due to curiosity, however, certain factors like harsh parenting and early separation from his mother could have sustained it. Though some have suggested that elements of sexually deviant behaviour may form part of psychotic symptoms occurring in the context of schizophrenia ${ }^{7}$, none of the patient's sexual fantasies were revealed in his delusions During the illness, the patient gradually withdrew from household activities preferring outdoor time with goats. Mr. A's increasing intimacy with animals while withdrawing from his family members might be attributable to the "friendly and nonjudgmental" nature of animals. ${ }^{6}$

Schizophrenia and sex offending may be mediated by several factors likely to be relevant in creating 
circumstances that are associated with committing a specific sex offence at a particular time. Certain psychosexual variables hypothesized to be associated with sex offending, such as cognitive distortions, lack of empathy, deficient social skills and deviant sexual fantasy, were found in this case. ${ }^{8}$ This patient's inability to conform to social norms might have been limited by ego disintegration arising from the illness. Schizophrenia may exacerbate deviant behavior on account of its effects on thoughts and urges, or the influence of delusions or hallucinations.

Premorbid zoophilic orientation and antisocial personality traits are two factors that might have mediated reoffending in the presented case. This is consistent with earlier studies suggesting that a history of psychosis, deviant sexual arousal, personality disorder, prior sexual offences, and the number of previous transgressions are predictive of reoffending. ${ }^{9}$ Evaluating the risk of future wrong-doing in mentally ill sex offenders remains a difficult challenge for clinicians. ${ }^{10}$ Thus, further research exploring the potential risk of reoffending is needed. A better understanding of these factors would facilitate the development of comprehensive prevention and treatment strategies for individuals with Schizophrenia and comorbid sexual disorders.

G Amoo, O Abayomio, AA Olashore Neuropsychiatric Hospital Aro, Abeokuta, Nigeria

\section{References}

1. World Health Organization. The ICD-10 classification of Mental and Behavioural Disorders. Diagnostic criteria for Research, 1992. Geneva.

2. Sadock BJ, Sadock VA. Human Sexuality. In Kaplan and Sadock's Synopsis of Psychiatry, 8th ed. Philadelphia: Lippincott Williams and Wilkins, 2003: 718-728.

3. Drake $C R$, Pathe M. Understanding sexual offending in schizophrenia. Criminal Behavioural and Mental health 2004; 14(2):108-120.

4. Earls MC, Lalumiere LM. A case study of preferential Bestiality. Archives of Sexual Behaviour 2009; 38(4): 605-609.

5. Earls MC, Lalumiere LM. A case study of preferential Bestiality. Sexual Abuse: A Journal of Research and Treatment 2007; 14(1): 83-88.

6. Williams CJ, Weinberg MS, . Zoophilia in Men: A Study of Sexual Interest in Animals. Archives of Sexual Behaviour 2003; 32(6): 523-35.

7. Smith AD, Taylor PJ. Serious sex offending against women by men with schizophrenia: Relationship of illness and psychotic symptoms to offending. British Journal of Psychiatry 1999; 174: 133-137.

8. Chesterman P, Sahota K. Mentally ill sex offenders in a regional secure unit. I: Psychopathology and motivation. Journal of Forensic Psychiatry 9 (1);1998; 150-160

9. Hanson K, Bussiere M. Predictors of Sexual Offender Recidivism: A meta-analysis. Ottawa, Ontario, Canada: Department of the Solicitor General Canada, User report 1996-04

10. O'Shaughnessy RJ. Commentary: Sex Offenders and Insanity. Journal of American Academic Psychiatry Law 2007; 35:4:451-453.

\section{(1) IACAPAP Adolescent Psychiatry and Allied Professions \\ IACAPAP TEXTBOOK OF CHILD \& ADOLESCENT MENTAL HEALTH"}

The "IACAPAP Textbook of Child and Adolescent Mental Health" is now available at the IACAPAP website http://iacapap.org/iacapap-textbook-of-child-and-adolescent-mental-health

\section{Please note that this book is:}

- Free and no registration is required to read or download it

- This is an open-access publication under the Creative Commons Attribution Non-commercial License. According to this, use, distribution and reproduction in any medium are allowed without prior permission provided the original work is properly cited and the use is non-commercial.

Please, inform colleagues, libraries and professionals who may be interested of the availability of this resource, provide feedback about the book, and encourage professional journals to review it. 